\title{
Corridor transeuropéen de transport et opposition à un projet d'aménagement : le chantier contesté de la gare de Stuttgart
}

Trans-European transport corridor and opposition to a development project: The contested development of Stuttgart railway station

Transeuropäische Verkehrskorridor und Widerstand gegen eine Städtebau: Das umstrittene Bauprojekt des Stuttgarter Bahnhofs

\section{Anaïs Volin}

\section{OpenEdition}

\section{Journals}

Electronic version

URL: http://journals.openedition.org/rge/5578

ISSN: $2108-6478$

Publisher

Association des géographes de l'Est

\section{Printed version}

Date of publication: 1 October 2015

ISSN: 0035-3213

Electronic reference

Anaïs Volin, «Corridor transeuropéen de transport et opposition à un projet d'aménagement : le chantier contesté de la gare de Stuttgart », Revue Géographique de l'Est [Online], vol.55 / n³-4 | 2015, Online since 13 October 2015, connection on 08 September 2020. URL : http:// journals.openedition.org/rge/5578

This text was automatically generated on 8 September 2020

Tous droits réservés 


\section{Corridor transeuropéen de transport et opposition à un projet d'aménagement : le chantier contesté de la gare de Stuttgart}

Trans-European transport corridor and opposition to a development project: The contested development of Stuttgart railway station

Transeuropäische Verkehrskorridor und Widerstand gegen eine Städtebau: Das umstrittene Bauprojekt des Stuttgarter Bahnhofs

Anaïs Volin

1 La thématique des réseaux transeuropéens de transport (RTE-T) n'est pas en soi un nouvel objet géographique puisque l'ouvrage de Roger Brunet (Brunet, 1991) introduisait déjà la notion de RTE-T. Cette dernière figurait dès 1990 dans le Rapport sur le réseau ferroviaire à grande vitesse rédigé par un groupe d'experts mandaté par le Conseil de l'Union européenne (Conseil de l'Union européenne, 1990). Le traité de Maastricht acte la mise en place des « Réseaux Transeuropéens de Transports, Energies et Communication » définis par l'Union européenne (UE). La littérature francophone et anglophone sur le sujet s'est développée dans les années 1990 parallèlement à la mise en place des quatorze premiers RTE-T. Les géographes se sont intéressés à la question des réseaux servant à relier des espaces éloignés en Europe (Damien, 1999 ; Cinotti, Tréboul, 2000 ; Varlet, 2000 ; Caron, Auphan, 2008 ; Debrie, Comtois, 2010) tandis que d'autres chercheurs (en économie et en géographie) se sont attachés, pour leur part, à montrer la valeur ajoutée économique de tels programmes européens dans la mesure où les échanges seraient facilités (Sichelschmidt, 1999; Dubois, 2010). Le géographe allemand Bernd Scholl (Scholl, 2014) renouvelle la question de la gouvernance des infrastructures de transport en Europe en étudiant le long processus de mise en place des projets d'aménagement (projet prioritaire $n^{\circ} 24$ : Rotterdam-Gênes) et la nécessité de coordonner les acteurs prenant les décisions et les acteurs mettant en œuvre les projets. À notre tour, nous revenons sur la longue et complexe mise en place des 
réseaux transeuropéens développés sous la forme de grands projets prioritaires puis de corridors transeuropéens de transport.

Cet article a pour objectif de montrer les difficultés auxquelles font face les acteurs politiques pour mener à bien un projet d'aménagement urbain lorsque les enjeux d'infrastructure dépassent l'échelle de réalisation du projet. Nous expliciterons la complexité du processus d'établissement d'un grand projet prioritaire européen à travers le cas de l'axe ferroviaire à grande vitesse Paris-Bratislava. Nous montrerons comment le grand nombre d'acteurs impliqués dans les processus de décision contribue à ralentir la mise en place d'un projet à l'échelle européenne. À cela vient s'ajouter la prise en compte de la société civile et de son engagement politique dans les grands projets d'aménagements. Quelles sont les échelles d'actions des mouvements d'opposition? Pouvons-nous envisager la constitution d'un mouvement contestataire national, voire européen? Nous analyserons simultanément l'émergence à plusieurs échelles de ce projet (Union européenne, Etat, région) ainsi que la multitude d'acteurs en présence (publics, privés et associatifs). Comment et pourquoi les pouvoirs publics (locaux, nationaux et européens) peuvent et doivent justifier ensemble la réalisation d'une infrastructure pourtant désavouée ? Dans quelle mesure pouvons-nous constater, à Stuttgart, une convergence des intérêts défendus par l'UE et par les acteurs locaux ? Un premier temps sera consacré au contexte européen dans lequel a été mise en place la ligne à grande vitesse Paris-Bratislava. Dans un second temps, nous nous intéresserons au projet d'aménagement, Stuttgart 21 comme un maillon du tronçon Stuttgart-Ulm soutenu par les acteurs locaux, nationaux et européens. Finalement, nous étudierons l'opposition à Stuttgart 21 ainsi que le contre-point pris par les acteurs porteurs du projet pour légitimer les travaux en cours.

3 Les recherches menées pour cet article font appel à un entretien effectué en décembre 2013 au sein de la "Direction mobilité et transport » de la Commission européenne; ainsi qu'un travail de terrain effectué à Stuttgart en 2014 et 2015 mêlant observations et entretiens auprès d'acteurs institutionnels. Nous avons ainsi pu rencontrer des membres du Ministère des Transports et des Infrastructures (MVI) du Land du BadeWurtemberg (BW), des acteurs institutionnels de la ville de Stuttgart ainsi que des universitaires allemands (urbanistes et politistes).

\section{Elaboration de la ligne ferroviaire à grande vitesse Paris-Bratislava : la gouvernance européenne des grands projets d'aménagement}

Les arguments développés dans cette première partie reviennent sur le contexte européen d'aménagement des territoires par les transports. Il semblerait que sans l'appui de l'échelle européenne et de sa politique des transports offensive sur la question ferroviaire, le projet de rénovation de gare à Stuttgart n'aurait pas été possible. 


\section{A. La mise en place des projets prioritaires européens entre 1994 et 2004 : un nécessaire quadrillage de l'Europe par les transports}

Les réseaux transeuropéens de transport (RTE-T) ont été développés au début des années 1990 et se sont institutionnalisés avec la signature du traité de Maastricht en 1992 dans les domaines du transport, des télécommunications et de l'énergie. Cette décision de la Commission européenne fait suite à l'établissement en 1992 d'un schéma unique pour la politique commune des transports décrit dans le Livre blanc des transports (Commission européenne, 1992). Il s'agit de favoriser " l'interconnexion et l'interopérabilité des réseaux nationaux ainsi que l'accès à ces réseaux" (Union européenne, 1992). L'UE souhaite développer des réseaux intermodaux (rail, route, transport fluvial) et unifiés (infrastructures et matériels similaires) à travers la mise en place de quatorze projets dits projets d'Essen ${ }^{1}$. Les modes de transport respectueux de l'environnement (transport ferroviaire et fluvial) sont privilégiés tout en cherchant à réduire les temps de transport entre les différentes villes. Le maillage des RTE-T a été complété en 2004 par seize autres projets prioritaires (Mayet, 2005 ; Degryse, 2007), désormais au nombre de trente et regroupés sous le nom de projets prioritaires déclarés d'intérêt européen (décision 884/2004).

6 Un projet prioritaire (Commission européenne, 2005) est un axe de transport (multimodal) qui traverse l'UE. Le transport ferroviaire reste le mode privilégié pour la création ou l'extension des axes ${ }^{2}$. Avec les trente projets prioritaires, les RTE-T dépassent le simple cadre de l'UE à quinze et s'étendent aux pays nouvellement entrés dans l'UE en 2004 ainsi qu'aux candidats à l'élargissement. L'enjeu de ces axes transeuropéens repose sur la volonté de renforcement des liens entre l'Europe de l'Ouest -pays membres de l'UE- et l'Europe de l'Est -pays nouvellement membres de l'UE ou en passe de le devenir- (Fontaine Vive, 2004). Ainsi, la mise en place des RTE-T participe à l'européanisation des territoires. Ce processus, abondamment étudié par les géographes (Bafoil, Beichelt, 2008 ; Rey, Groza, 2008 ; Lepesant, 2011), peut être défini comme « un processus de construction territoriale, institutionnelle et de valeurs, orienté vers une certaine convergence d'échelle macro » (Rey, Groza, 2008) et peut s'appliquer aux trente projets prioritaires européens.

7 Le financement est assuré en partie par les fonds européens (Commission européenne, 2005) avec le budget RTET-T, le fonds de cohésion, le Fond européen de développement économique régional, l'Instrument d'aide à la pré-adhésion et la Banque européenne d'investissement ainsi que par la contribution directe des États. En ce qui concerne les fonds privés, d'éventuels partenariats public-privés peuvent être établis et financer une partie des travaux. Chaque projet selon sa nature (projets transfrontaliers, projets avec goulet d'étranglement ou autres) est soumis à un taux maximal de soutien de la part des fonds de l'UE ${ }^{3}$. Les coûts de chaque projet prioritaire sont réévalués chaque année avec la possibilité de voir les dépenses augmenter dans un contexte de crise budgétaire. Les projets prioritaires ne sont cependant pas les seuls outils développés par l'UE pour concourir à étendre ses réseaux de transport, les corridors transeuropéens constituent le second volet de la politique européenne des transports. 


\section{B. Des projets prioritaires européens aux corridors transeuropéens : vers un nouveau maillage de l'espace européen}

Un corridor est un «axe multimodal raccordant le RTE-T avec les principales infrastructures dans les pays d'Europe Centrale et Orientale afin d'intensifier la coopération et préparer les pays candidats à leur future adhésion à l'Union " (Siarov, 2004). En 1994, lors de la 2ème conférence paneuropéenne des transports en Crète, neuf corridors sont définis par la Commission européenne puis un 10ème corridor est créé en 1997 lors de la 3ème Conférence paneuropéenne des transports d'Helsinki. Nous avons ainsi deux réseaux complexes qui manquent d'interconnexions et posent la question des doublons au niveau de la gouvernance des infrastructures de transport et des fonds investis dans ces deux réseaux. Devant la nécessité de rassembler tous ces projets en une seule entité afin de simplifier le maillage existant et face aux difficultés financières des États et de l'UE, la Commission européenne a décidé fin 2013 de constituer un seul réseau (Figure 1) (Commission européenne, 2014). La promotion des neuf nouveaux corridors transeuropéens est assurée par la diffusion d'une vidéo (Commission européenne, 2013a) insistant sur « le passage d'un maillage dispersé à un réseau resserré $\rrbracket^{4}$. Chaque corridor associe au minimum trois modes de transport, trois États membres de l'UE et deux sections transfrontalières. Le réseau central devra être achevé en 2030 puis un réseau secondaire suivra d'ici 2050. Ce réseau secondaire viendra compléter les principaux axes par des liaisons internes aux pays.

Figure 1 : Historique de la mise en place des projets prioritaires et des corridors paneuropéens

\begin{tabular}{|c|c|c|}
\hline Dates & \multicolumn{2}{|c|}{ Projets } \\
\hline 1994 & $\begin{array}{l}14 \text { projets } \\
\text { prioritaires }\end{array}$ & $\begin{array}{l}9 \text { corridors } \\
\text { paneuropéens }\end{array}$ \\
\hline 1997 & & $\begin{array}{l}10 \text { corridors } \\
\text { paneuropéens }\end{array}$ \\
\hline 2004 & $\begin{array}{l}30 \text { projets } \\
\text { prioritaires }\end{array}$ & \\
\hline 2014 & 9 corrid & uropéens \\
\hline
\end{tabular}

L'UE possède des outils afin de développer ses réseaux de transports, réseaux qu'elle finance en partie par l'intermédiaire des fonds européens qui allouent 26 milliards d'euros (programmation 2014-2020) pour le financement des nouveaux corridors transeuropéens au titre du "Mécanisme pour l'interconnexion en Europe" (Commission européenne, 2014). Une partie de ces fonds sert à financer la ligne 
ferroviaire Paris-Bratislava, ce qui marque la volonté d'intensifier la connexion des États européens.

\section{C. «Un des axes clés reliant l'Europe de l'Ouest et l'Europe de l'Est ${ }^{5}$ : la ligne ferroviaire transeuropéenne Paris-Bratislava}

La ligne Paris-Bratislava figure parmi les trente projets prioritaires établis en 2004 et porte le numéro 17. Elle consiste en la construction et la modernisation de la ligne ferroviaire qui s'étend sur $1254 \mathrm{~km}$. Il s'agit de relier les deux capitales via l'Allemagne et l'Autriche (Figure 2) à travers des espaces urbains densément peuplés ${ }^{6}$ comprenant des capitales d'État et de région: Strasbourg pour l'Alsace, Stuttgart pour le BW, Munich pour la Bavière, Salzbourg pour le Land éponyme et Linz pour le Land oberösterreich.

Figure 2 : Tracé du projet prioritaire $\mathrm{n}^{\circ} 17$ Paris-Bratislava : une nouvelle ligne ferroviaire transeuropéenne

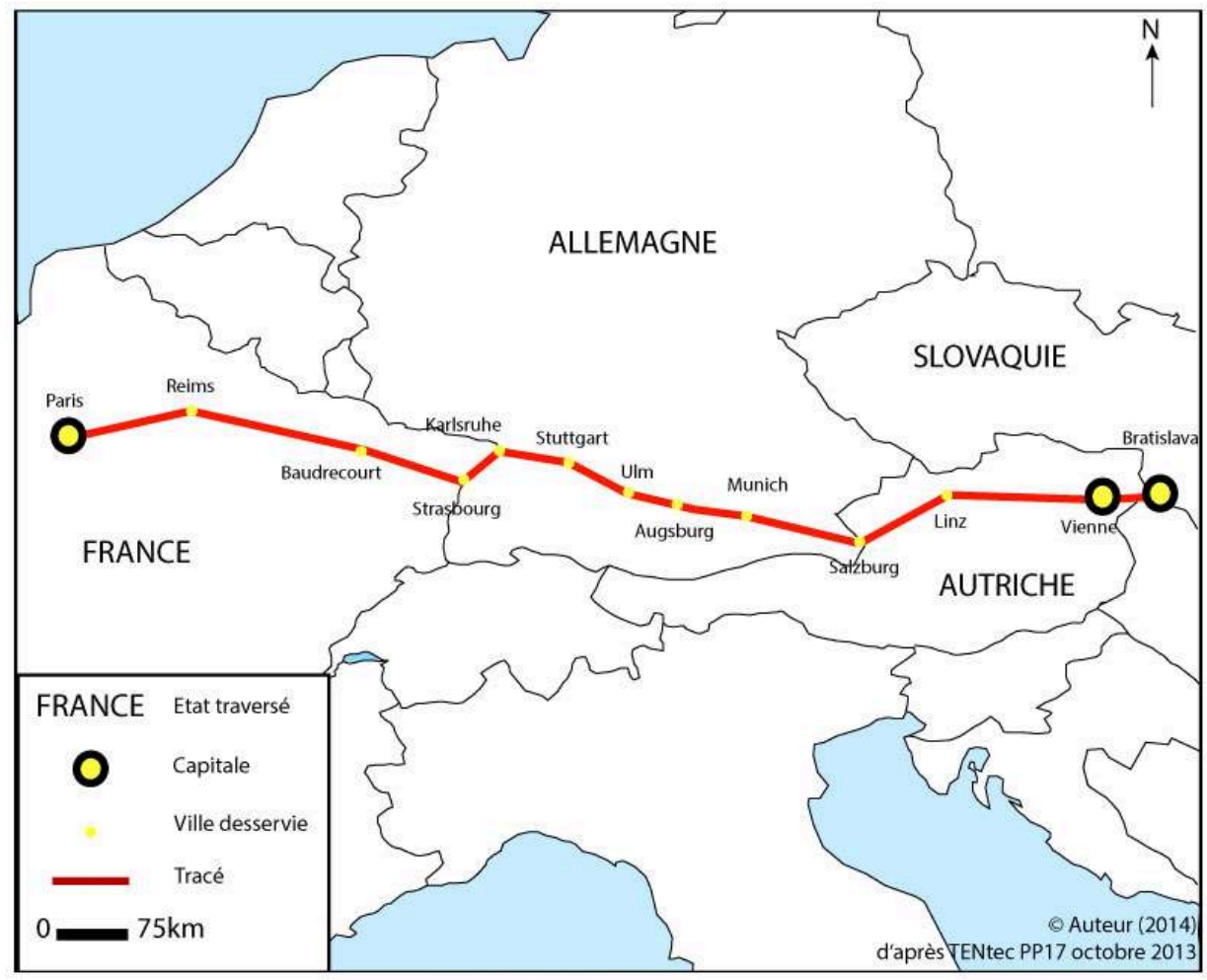

11 La volonté de mettre en place cette ligne ferroviaire est née de la nécessité de réduire le temps de transport entre les villes prenant part au projet, notamment par le passage à la grande vitesse. À terme, le trajet devrait durer environ $8 \mathrm{~h}$ contre 12 actuellement. Péter Balázs, ancien commissaire européen hongrois, fut coordinateur de l'axe entre juillet 2005 et décembre 2013ㄱ. Le coût total était estimé en 2006 à 23 milliards d'euros (Balázs, 2007) pour l'ensemble des travaux le long de la ligne. Les fonds dédiés à cette

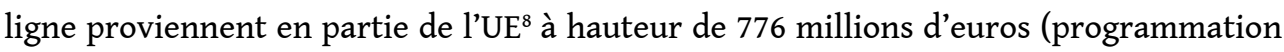
1995-2015) et en partie des États et des collectivités. La mise en place de ce projet a été permise grâce à l'accord des quatre pays concernés (France, Allemagne, Autriche, Slovaquie). Le projet ne pouvait, en effet, se faire qu'à condition que le tracé figure déjà dans les plans nationaux des transports des quatre États. L'achèvement des travaux est 
prévu, pour le moment, à l'horizon 2020/2021. Lors de la refonte de la carte des projets européens de transport en 2014, le projet prioritaire $n^{\circ} 17$ Paris-Bratislava s'est scindé en deux (Commission européenne, 2013b). Désormais la section Paris-Strasbourg intègre le corridor Atlantique coordonné par Karla Peijs (Pays-Bas) et la section Strasbourg-Bratislava rejoint le corridor Rhin-Danube coordonné par Carlo Secchi (Italie). La question est donc posée de savoir ce qu'il advient à présent de la ligne ParisBratislava. Le projet perdure par l'intermédiaire de l'association Magistrale pour l'Europe ${ }^{9}$. L'auteure poursuit actuellement ces recherches à ce sujet.

Le long de cette ligne Paris-Bratislava, des tronçons avaient été identifiés comme devant être effectués en priorité, qu'il s'agisse de section transfrontalière ou de goulet d'étranglement (Balázs, 2008). Les goulets d'étranglement correspondent à des espaces saturés comprenant la section Baudrecourt-Vendenheim en France, Stuttgart-Ulm en Allemagne et St-Pölten-Vienne en Autriche. Les espaces transfrontaliers concernés sont: Strasbourg-Kehl-Appenweier, Munich-Salzbourg et Vienne-Bratislava. Dans son dernier bilan de 2013 Péter Balázs revient sur l'épineuse question du financement des projets dans la mesure où les coûts estimés ont dû être revus à la hausse. C'est pourquoi au sein de chaque axe une hiérarchie des projets a été mise en place dans le but d'effectuer en premier lieu les travaux prioritaires (goulet d'étranglement ou section transfrontalière). Le goulet d'étranglement représenté par la section Stuttgart-Ulm ne revêt pas seulement une importance pour l'amélioration du réseau entre Paris et Bratislava ; mais constitue également un véritable enjeu présent depuis les années 1980 pour les acteurs aux échelles nationales, régionales et locales. La réalisation d'un nouveau nœud de transport à Stuttgart permettrait d'améliorer les transports régionaux et nationaux par l'intermédiaire de la ligne Mannheim-Munich. Sans le soutien financier et politique à ces différentes échelles, la nouvelle ligne ferroviaire entre Stuttgart et Ulm n'aurait pas pu voir le jour.

\section{L'arrivée d'une nouvelle ligne régionale Stuttgart- Ulm dédiée à la grande vitesse : plus de capacité avec le nouveau tracé}

13 L'étude régionale et locale effectuée ici sur la ligne Stuttgart-Ulm fait donc partie intégrante de la future ligne à grande vitesse transeuropéenne Paris-Bratislava.

\section{A. La ligne ferroviaire Stuttgart-Ulm avant le projet prioritaire européen}

La ligne Stuttgart-Ulm est une ligne ferroviaire construite entre 1845 et 1850 sous le règne de Guillaume 1er, roi du Royaume de Wurtemberg. Cette ligne historique, nommée Voie orientale (Ostbahn) ou Voie de la vallée du Fils ${ }^{10}$ (Filstalbahn), était la première à traverser le Royaume du Wurtemberg sur environ $93 \mathrm{~km}$. La ligne électrifiée en 1933 était alors la plus utilisée en République Fédérale Allemande avec un maximum de 430 trains par jour par jour en 1970 (Heuschele, 1970). En 1985, le ministère fédéral des transports établit un plan général des transports afin d'améliorer, entre-autres, les liaisons ferroviaires existantes. La ligne à grande vitesse Mannheim-KarlsruheStuttgart-Ulm-Munich est une des lignes prioritaires. Pour la section Stuttgart-Ulm 
différents tracés sont envisagés dès 1988 avant que le projet soit officiellement présenté en $1994^{11}$. L'actuelle voie (trains passagers et marchandises) entre Stuttgart et Ulm suit le Fils (passage par le Nord) tandis que le projet de nouvelle ligne à grande vitesse passera par le sud et suivra l'autoroute A8. (Figure 3)

Figure 3 : Le projet ferroviaire Stuttgart-Ulm : un nouveau tracé en faveur de la grande vitesse

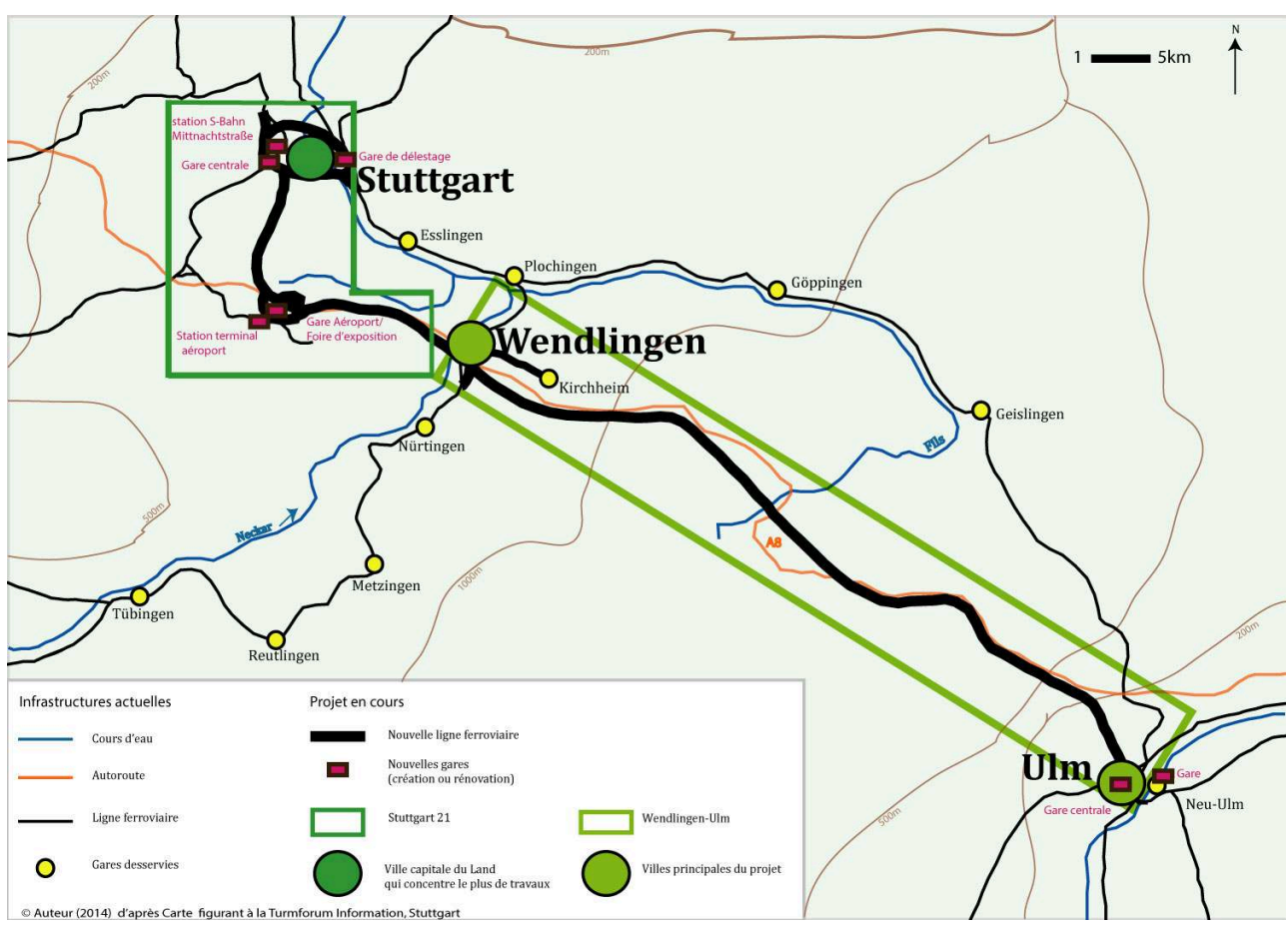

Des travaux étaient nécessaires sur cette ligne Stuttgart-Ulm, décrite comme «la section la plus compliquée » (Balázs, 2008) sur l'ensemble du tracé Paris-Bratislava. Le projet de nouvelle ligne, long de $121 \mathrm{~km}$, permettrait de désengorger la ligne actuellement saturée. Quelles sont les modalités des travaux envisagés? Quels aménagements urbains sont prévus?

\section{B. Le projet Stuttgart 21 : enjeu central des travaux}

La signature d'un accord en 2007 entre le ministère fédéral allemand des transports, la Deutsche Bahn et les collectivités locales (le maire de Stuttgart, le ministre-président $\mathrm{du}$ Land ainsi que le ministère des transports du Land) constitue la première pierre de lancement des travaux sur la ligne Stuttgart-Ulm. Les travaux ont commencé à Stuttgart en 2010 et le tracé devrait être opérationnel d'ici à 2021 (Balázs, 2013). Le projet ferroviaire Stuttgart-Ulm se décompose en deux sous-projets: Stuttgart 21 (travaux dans la ville et section entre la ville et l'aéroport) et la section WendlingenUlm (Balázs, 2008) dont le coût total ${ }^{12}$ était estimé à 7,4 milliards d'euros en 2010 et à 9,8 milliards d'euros en mars 2013. Les acteurs de ces projets se sont regroupés dans un consortium en 2009 appelé « Union pour le projet ferroviaire Stuttgart-Ulm » (Verein Bahnprojekt Stuttgart-Ulm) centralisant les principaux partenaires publics et privés. Les membres de cette association d'intérêts sont la Deutsche Bahn ${ }^{13}$, le Land du BadeWurtemberg, la ville-capitale du Land Stuttgart, la région métropolitaine de Stuttgart (Verband Region Stuttgart), l'aéroport de Stuttgart et la ville d'Ulm. 
Le projet Stuttgart 21, synonyme de Stuttgart pour le XXIème siècle, comprend deux aspects (Figure 3): d'une part la rénovation de la gare centrale de Stuttgart avec la transformation de l'actuelle gare terminus (Kopfbahnhof) en une gare traversante (Durchgangsbahnhof) ainsi que des travaux annexes [nouvelle gare de train express régional (S-Bahn) et nouvelle gare de dépôt], d'autre part la construction d'une nouvelle gare multimodale dans l'aéroport de Stuttgart, situé à Filderstadt, afin que l'aéroport soit plus accessible depuis Stuttgart et depuis Ulm. Le temps de trajet prévu entre Stuttgart et l'aéroport est de 8 minutes $^{14}$ au lieu de 27 actuellement en S-Bahn et de 29 minutes entre Ulm et l'aéroport au lieu d'environ $1 \mathrm{~h} 40^{15}$ actuellement (Brochure Bahnprojekt Stuttgart-Ulm, 2014). Lors du lancement des travaux dans la gare de Stuttgart, des écriteaux (Photo 1) ont été affichés sur les panneaux se trouvant sur les quais. Cette communication est à l'initiative du bureau de communication de l' "Union pour le projet ferroviaire Stuttgart-Ulm ${ }^{16}$ dans le but d'informer les habitants de la ville et les usagers de la gare de Stuttgart. Il était indiqué : "C'est parti! Le projet Stuttgart 21 arrive. Début des travaux le 2 février $2010 »^{17}$. La gare centrale se trouvant au cœur du centre-ville, à l'extrémité de la Theodore-Heuss Straße (artère centrale de la ville), la question de l'accessibilité pendant les travaux est primordiale et explique, en partie, le dispositif de communication mis en place.

Photo 1 : Lancement des travaux Stuttgart 21

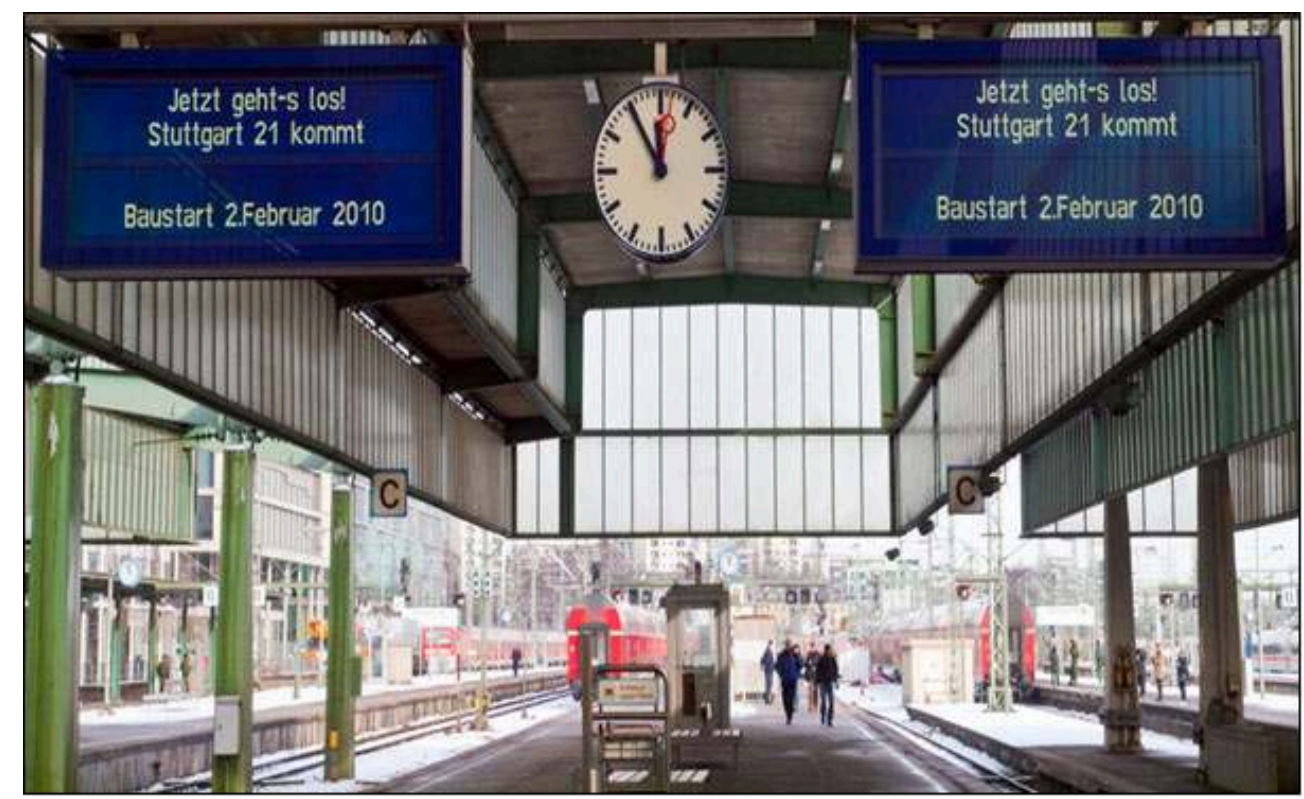

Source : Bureau de communication Bahnprojekt Stuttgart-Ulm, 2010

Stuttgart est la capitale du Land du BW, Land traditionnellement dirigé par la $\mathrm{CDU}^{18}$ mais qui a connu le passage d'une coalition $\mathrm{CDU}^{-S D^{19}}{ }^{19}$ ou CDU-FDP ${ }^{20}$ à une majorité Die Grünen ${ }^{21}-\mathrm{SPD}^{22}$. Le Land depuis 2011 et la ville-capitale de Stuttgart depuis 2014 sont ainsi dirigés par une coalition Die Grünen-SPD. La coalition CDU-SPD au pouvoir dans les années 1990 projetait avec la Deutsche Bahn de repenser la gare et le quartier situé au nord, dit Nordbahnhofviertel, situé entre le parc Rosenstein et la gare centrale. La nouvelle majorité en place Die Grünen-SPD, a été élue en 2011, après la fixation des plans et le début des travaux, ce qui leur laisse peu de marge de manœuvre. La première présentation du projet a eu lieu en 1994 par la Deutsche Bahn, le Land du 
BW et la ville de Stuttgart. Il s'en suit une dizaine d'années pour établir le plan financier et la programmation des travaux. Ils incluent le passage des voies ferrées en souterrain ainsi que l'aménagement de deux quartiers attenants: Europaviertel et Rosensteinviertel.

Ce projet initié dès les années 1980 est à présent entre les mains d'une coalition politique dans la ville de Stuttgart et dans le Land qui, pour la majorité, était défavorable au projet. Les travaux commencés avant l'élection du nouveau parlement régional continuent mais la communication avec les citoyens semble restaurée, notamment grâce au référendum organisé en 2011 dans le Land. Une majorité des habitants s'est prononcée en faveur de la poursuite du financement de Stuttgart 21 par le Land, et cela malgré un coût des travaux élevé.

Le coût total des travaux pour Stuttgart 21 était de 6.5 milliards d'euros en 2013 (Bahnprojekt Stuttgart-Ulm, 2014) financés de la manière suivante (Figure 4),

- 57\% par la Deutsche Bahn ${ }^{23}$

- 19\% par l'Etat fédéral allemand et l'Union européenne

- 14\% par le Land du BW

- 5\% par la ville-capitale Stuttgart

- 4\% par l'aéroport de Stuttgart

- 1\% par la région métropolitaine de Stuttgart

Figure 4 : Financement du projet ferroviaire Stuttgart-Ulm

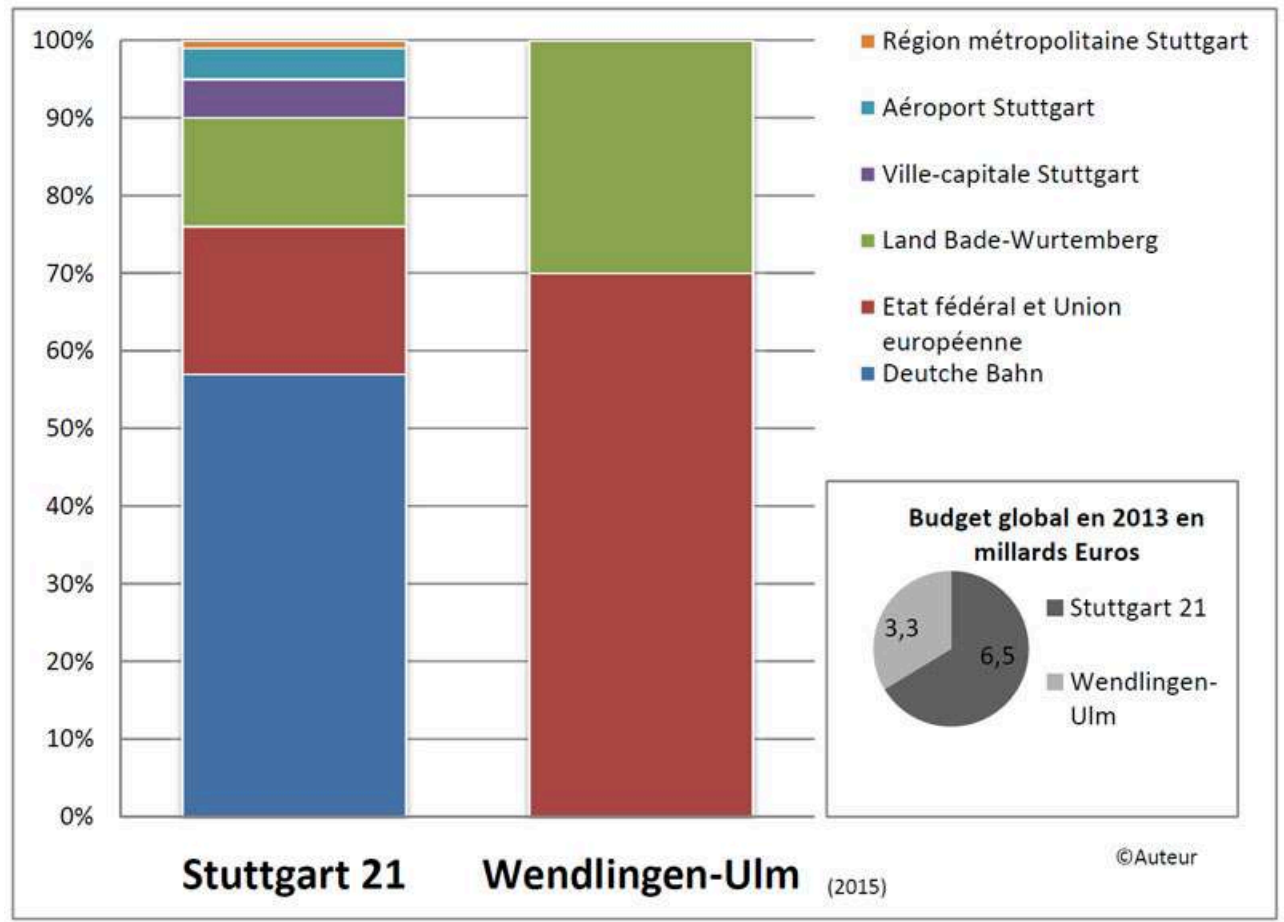

21 Le projet Stuttgart 21 s'étend également à l'espace de l'aéroport avec la création d'une nouvelle gare ferroviaire. Enfin, deux autres gares font partie du projet à savoir la nouvelle station de S-Bahn Mittnochstraße qui verra ainsi le point nodal du réseau de S-Bahn déplacé de la gare centrale vers cette gare (au nord de la gare centrale). Une nouvelle gare de délestage est également prévue dans les plans sur l'ancienne ligne Stuttgart-Ulm située dans le quartier Untertürkheim (Figure 3). 
Le projet Wendlingen-Ulm, quant à lui, consiste en la création d'une ligne ferroviaire à grande vitess ${ }^{24}$ sur $60 \mathrm{~km}$ dont 31 en tunnels en raison du relief (Jura Souabe). Le financement des dépenses, estimées à 3,3 milliards d'euros en 2013 (Bahnprojekt Stuttgart-Ulm, 2014) se fait à hauteur de 30\% par le Land du BW et de $70 \%$ par l'Etat fédéral allemand et l'UE. Cette section est donc en majorité financée par l'Etat fédéral et l'UE (Figure 4). Les échelles nationales et européennes soutiennent ces deux projets dont la mise en place coïncide avec la création des grands projets d'aménagement européens dans les années 1990 et 2000. Bien que le projet ait été initié par la Deutsche Bahn et la ville de Stuttgart, le Bund par l'intermédiaire du ministère fédéral des transports soutient le projet Stuttgart 21 en tant que grand projet d'infrastructure.

Quels sont les motifs justifiant la réalisation de ces grands travaux? Comment les acteurs européens, nationaux et locaux se soutiennent dans leurs politiques d'aménagement?

\section{Les arguments en faveur d'une nouvelle ligne ferroviaire à grande vitesse entre Stuttgart et Ulm : aménagements ferroviaires et projets urbains}

Les soutiens au projet de nouvelle ligne à grande vitesse ferroviaire entre Stuttgart et Ulm (faire la distinction entre les travaux autour de la gare de Stuttgart et la nouvelle ligne à grande vitesse) ne manquent pas, en particulier de la part des acteurs qui en sont à l'origine ${ }^{25}$ mais aussi de la part des acteurs européens. Les porteurs du projet nationaux, régionaux et locaux, avec le soutien de l'UE ont présenté des arguments généraux contribuant à l'amélioration du réseau ferré dans l'ensemble du Land mais également des arguments en faveur de la ville de Stuttgart pour certains, d'Ulm pour d'autres. Ces différents arguments prennent place dans des cadres spatiaux allant de l'échelle européenne à l'échelle locale.

Les acteurs publics et privés en faveur de la nouvelle ligne à grande vitesse entre Stuttgart et Ulm arguent que cette ligne concourt à une meilleure liaison entre l'Est et l'Ouest à l'échelle européenne. Il s'agit de promouvoir les transports dits durables, moins polluants que le transport routier et aérien, à la suite des recommandations inscrites dans le Livre blanc des transports (Commission européenne, 2011b). À l'échelle macro-régionale et nationale la nouvelle ligne à grande vitesse (jusqu'à $250 \mathrm{~km} / \mathrm{h}$ ) permettra de relier plus rapidement Stuttgart à Munich ( $1 \mathrm{~h} 37$ au lieu de 2 h10) ou Vienne (4 h 50 au lieu de 7 h) (Jünemann, 2006) ce qui permet d'entrer en concurrence avec l'avion. L'incitation à la création de liaisons rapides entre les villes sert la promotion du renforcement des liens entre les villes dans un espace supranational (fer de lance de l'UE). De même, ce projet participe à la mise en compétition des territoires métropolitains avec un nouvel enjeu qui va être celui de devenir la ville la plus attractive de l'aire géographique et se faire une place parmi les métropoles reconnues d'Europe occidentale, toutes les échelle s'entrecroisent ici (du local à l'européen).

26 À l'échelle régionale, les temps de trajet à l'intérieur du BW seront écourtés (Figure 5), ce qui va permettre de relancer le marché du transport ferroviaire dans un Land où l'automobile est reine ${ }^{26}$. Deux trajets attirent particulièrement notre attention, celui entre Ulm et l'aéroport et celui entre Stuttgart et l'aéroport (Figure 3). Les deux villes voient leur temps de trajet à l'aéroport au minimum divisé par deux, ce qui est un argument important pour l'attractivité économique de ces deux villes. 
Figure 5 : Temps de trajet actuels et à venir entre différentes villes du Land du BW

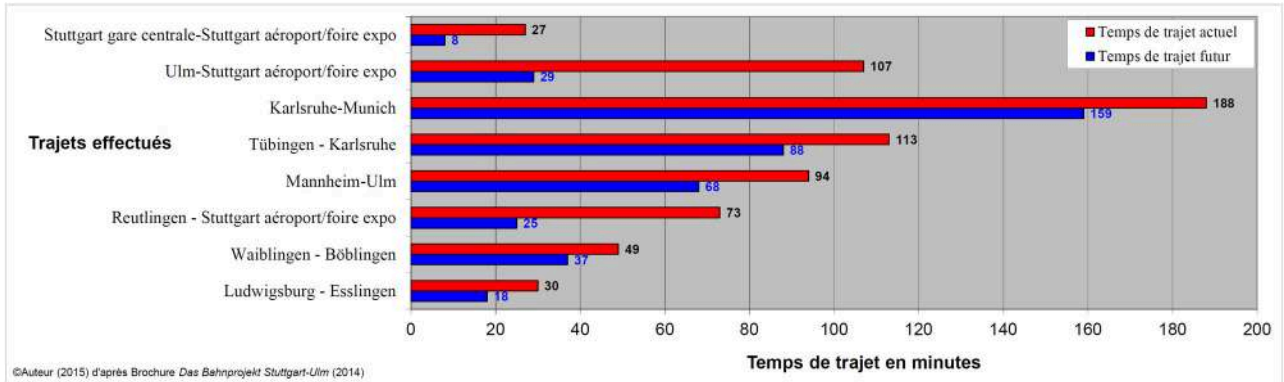

Source : Brochure Bahnprojekt Stuttgart-UIm, 2015 prennent place dans les nouveaux quartiers jouxtant la gare (Europaviertel et Rosensteinviertel). Le quartier Europe est directement connecté à la gare centrale de Stuttgart et jouit ainsi d'une situation privilégiée pour accéder aux trains à courte et longue distance. L'accessibilité en train et en transport en commun est privilégiée avec le passage de la ligne de métro $\mathrm{n}^{\circ} 12$ et son arrêt Stadtbibliothek devant la nouvelle bibliothèque de la ville. Ce quartier accueille un ensemble d'immeubles de bureaux dont ceux de la Banque du Land du BW (Landesbank Baden-Württemberg), ce qui tend à faire de ce lieu un nouveau quartier d'affaire dans la capitale du Land. Le nom de ce quartier, quartier de l'Europe, peut rappeler le quartier français d'Euralille ou l'Europaviertel de Francfort. Il s'agit de la construction d'un quartier proche d'un hub de transport et comportant un quartier d'affaires au cœur d'une grande ville européenne.

Les acteurs politiques de Stuttgart ont également projeté de créer de nouveaux espaces verts (notamment dans la continuité du parc Rosenstein) et des logements en centreville en remplacement des voies de chemins de fer desservant la gare puisque celles-ci seront, à terme, enterrées. Les employés et les futurs résidants auront ainsi un accès privilégié à l'ensemble des aménités de la ville ${ }^{28}$. Ces projets semblent porteurs d'une nouvelle dynamique donnée à la ville face au manque de logements d'une part et d'autre part grâce à la volonté d'accueillir de nouvelles activités de tertiaire supérieur (bureaux). En effet, la ville souhaite attirer de nouvelles entreprises travaillant dans le secteur tertiaire afin de diversifier ses activités économiques. D’après une étude de 2013, dans la ville de Stuttgart, les trois premiers secteurs d'emploi sont la production de voitures, les services de santé et la construction de machines ${ }^{29}$. Cette ville n'est pas tournée en premier lieu vers les services, ce qui justifie le souhait de la part de la municipalité de construire de nouveaux quartiers dédiés au tertiaire. En parallèle, la demande de logements, notamment de la part des jeunes ménages mais aussi des plus 
anciens, continue d'être forte (Stuttgarter Zeitung, 24 septembre 2012; Stuttgarter Nachrichten, 6 décembre 2013), ce qui peut expliquer la volonté de faire d'Europaviertel et Rosensteinviertel des nouveaux quartiers d'habitation proches du centre. Les familles avec des jeunes enfants peinent encore à trouver des logements qui leur conviennent ${ }^{30}$, ce qui devient un véritable enjeu social et démographique pour la ville de Stuttgart. Ce projet de rénovation de quartier de gare n'est pas le seul sur la ligne Paris-Bratislava (Vienne, Bratislava) et semble répondre à des besoins locaux qui ne sont pas partagés par tous.

30 Nous allons tenter d'expliquer les raisons de cette opposition et comprendre qui sont les acteurs de la contestation. Pouvons-nous seulement parler de mouvement local? Comment les porteurs du projet se mobilisent-ils pour continuer à faire avancer les travaux?

\section{La construction multiscalaire d'une légitimation du projet Stuttgart 21 face aux contestations de la société civile}

\section{A. La contestation locale de Stuttgart 21 : le tournant de 2010}

31 Le projet Stuttgart 21, implique de longs travaux dans le centre-ville de Stuttgart ce qui influe, entre autres, sur les transports en commun. Le passage des voies ferrées de l'aérien au souterrain demande un réaménagement complet de la gare centrale et des quartiers alentours, ce qui fait l'objet de vives contestations de la part des citoyens. Dans les faits cela a commencé par des manifestations en novembre 2009, appelées «Manifestations du lundi » (Montagsdemo) puisque que les manifestants se retrouvent chaque lundi soir depuis cette date. Des heurts parfois violents ont eu lieu en 2010 entre les manifestants et la police avec l'épisode marquant de la manifestation du jeudi 30 septembre 2010 pendant laquelle les participants ont été aspergés d'eau et de gaz lacrymogène. Les manifestants sous l'égide, entre-autres, du mouvement des "défenseurs du parc» (Parkschutzer), étaient venus exprimer leur désaccord concernant la destruction d'une partie du parc attenant à la gare centrale. Malgré cela, l'attachement d'une partie des citoyens aux arbres centenaires du parc du château ${ }^{31}$ reste grand (Architektinnen K21, 2013). Cet événement a été largement relayé par les médias nationaux et internationaux sous le terme de "Jeudi noir» (Schwarzer Donnerstag) (Rue 89, 22 avril 2011) et le procès des acteurs politiques, ayant autorisé la répression de cette manifestation, se déroule depuis le 24 juin 2014 (Stuttgarter Zeitung, 10 septembre 2014 ; Stuttgarter Zeitung, 17 septembre 2014).

Les manifestants sont opposés au projet Stuttgart 21 pour diverses raisons, exposées dans plusieurs ouvrages et articles parus dès 2010 (Schorlau, 2010 ; Häußler, 2011 ; Kappus, 2011 ; Lösch et al., 2011 ; Reiser, 2011 ; Novy et Peters, 2012). La contestation porte d'une part sur le fond : À qui va profiter le passage de plus d'intercity express (ICE) alors que les habitants du Land utilisent d'abord leurs voitures pour leurs déplacements (Statistisches Landesamt, 2013) ${ }^{32}$ ? Quelles justifications sont avancées face à l'effort financier demandé (augmentation croissante des coûts) ? D'autre part sur la forme, le manque de consultation préalable au début des travaux apparaît comme une remise en cause des acteurs politiques anciennement au pouvoir (notamment Stefan Mappus, CDU, ancien président du Land du BW), la présidence du Land étant 
assurée depuis 2011 par Winfried Kretschmann du parti des Verts. Les arguments se focalisent, entre-autres, sur le manque de démocratie participative dans la mise en place des projets urbains. Les opposants se sont regroupés en associations, par exemple l' « Union Kopfbahnhof 21 » milite pour un projet alternatif. Il existe d'autres collectifs comme Oben bleiben, ArchitektInnen für K21, Parkschützer (Götz von Olenhusen, Paulus, 2010) dont certains comme ArchitektInnen für K21 sont composés de professionnels (architectes, urbanistes) (ArchitektInnen für K21, 2013). La présence d'opposants dès 1995 disposant d'une capacité d'expertise semble complexifier le rapport de force entre les porteurs de projets et ses détracteurs dans la mesure où ces derniers sont en capacité de proposer de véritables projets alternatifs tel Kopfbahnhof 21. Ce dernier argument nous montre que ces groupes d'opposition au projet, bien que locaux, ne peuvent être associés à des revendications NIMBY. Les différents collectifs investissent l'espace public par l'intermédiaire des «Manifestations du lundi » mais aussi par des campagnes d'affiches qui irriguent Stuttgart. Nous pouvons ainsi voir des affiches d'opposition au projet Stuttgart 21 dans la ville (Photo 2) et particulièrement près de la gare où se concentrent actuellement les travaux. Le collectif Kopfbahnhof 21 a repris le logo officiel en le barrant en signe d'opposition à Stuttgart 21.

Photo 2 : Logo symbole de l'opposition à Stuttgart 21

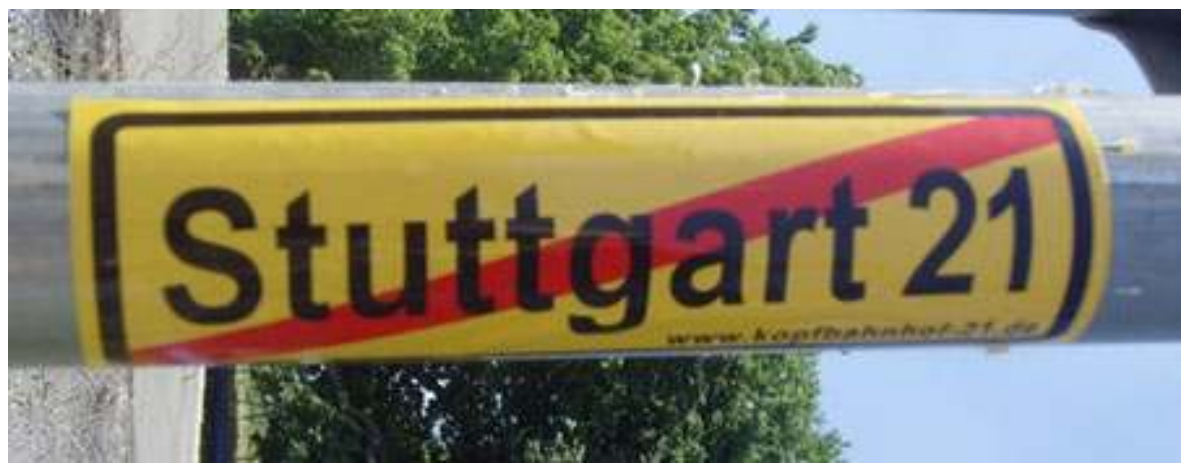

Source : ( ) Auteur, 2014

À la suite des altercations entre policiers et manifestants en septembre 2010, Heiner Geißler ${ }^{33}$ est nommé médiateur du projet en octobre 2010. Il propose un projet émanant de Stuttgart 21 dit "Stuttgart 21 plus» dans lequel, par exemple, seuls les arbres malades dans le parc du château seront abattus (Spiegel, 2010). Catherine Perron dans son article de 2011 (Perron, 2011) tente de comprendre «comment et pourquoi une affaire locale a pu enflammer de la sorte la ville et le Land provoquant ainsi les plus importantes manifestations jamais vues à Stuttgart». Le projet a pris, en effet, une envergure nationale, suite à l'ampleur prise par les manifestations, ce dont témoigne la prise de position en faveur du projet de la Chancelière Angela Merkel relayée par l'article du 23 novembre 2010 de Stefan Braun dans le Süddeutsche Zeitung (Süddeutsche Zeitung, 23 novembre 2010). Le projet reste soutenu par les acteurs nationaux du fait de leur engagement financier non négligeable ainsi que pour retrouver la confiance politique dans un Land perdu par la CDU au profit des verts.

Malgré la mobilisation des porteurs du projet, les "Manifestations du lundi» continuent (Deront, 2013), les retards sur le chantier s'accumulent et les arguments mis en exergue par les manifestants (coût élevé, destruction d'espaces verts, gênes 
occasionnées par les travaux) (Brun, 2013) trouvent un écho dans d'autres projets d'aménagement européens.

\section{B. Un conflit qui résonne à l'échelle transnationale : le réseau des Grands Projets Inutiles et Imposés (GPII)}

Stuttgart 21 est un exemple, parmi d'autres, de grand projet d'aménagement conflictuel en Europe. Les arguments économiques, environnementaux ou démocratiques soutenus par les opposants au projet Stuttgart 21 se retrouvent dans le cadre d'autres projets d'aménagement ferroviaire. Par exemple la ligne à grande vitesse Lyon-Marseille dans les années 1990, la construction de la ligne ferroviaire à grande vitesse entre Lyon et Turin (projet prioritaire européen) ou encore la ligne ferroviaire à grande vitesse traversant le Pays Basque. Cela nous montre la multiplication des aménagements ferroviaires à l'échelle européenne en même temps que l'émergence d'un engagement citoyen contre ces projets, dans un premier temps à l'échelle locale. Les travaux de Jacques Lolive et Stéphanie Leheis (Lolive, 1999 ; Leheis, 2012) posent, à cette occasion, la question de la participation citoyenne dans la mise en place de grands projets d'aménagement (Melé, 2008) et leur capacité à influer sur les décisions politiques locales et régionales. Les acteurs politiques du Land ont dû faire face à la mobilisation d'acteurs certes locaux mais aussi nationaux (implication du Bund für Umwelt und Naturschutz) et transnationaux avec les militants des «Grands Projets Inutiles et Imposés ».

Différents mouvements de contestation se sont regroupés en 2010 dans un collectif œuvrant contre les "Grands Projets Inutiles et Imposés» (GPII). Ce groupement a recensé à l'échelle mondiale l'ensemble des projets d'aménagement considérés comme Inutiles et Imposés, et les a cartographiés selon leur nature (projets ferroviaires, projets autoroutiers, forages pétroliers...). Ces projets «constituent pour les territoires concernés un désastre écologique, socio-économique et humain » d'après la Charte de Tunis $^{34}$ parue en 2013. Après le Val de Suze et Notre-Dame-des-Landes, le troisième forum annuel des GPII s'est déroulé en juillet 2013 à Stuttgart au cœur du Nordbahnhofviertel (Reporterre, 22 mai 2013), comme un message envoyé aux acteurs porteurs du projet pour montrer que la contestation devenait transnationale. Il semble que ce ne soit pas seulement un projet de rénovation de gare centrale, projet qui a pu se réaliser dans d'autres villes européennes (Delage, 2013) sans que les mouvements de contestation soient autant relayés. L'opposition ancrée à Stuttgart trouve des résonances dans d'autres espaces européens aux motifs de contestation proches. La dimension transeuropéenne conférée par les GPII à Stuttgart 21 permet, en réalité, de donner à entendre la voix de certains groupes dénonçant les grands projets d'aménagement européens. Nous pouvons nous demander si les GPII ne se sont pas associés à Stuttgart 21 pour en faire un fleuron de la dénonciation de la négation de la démocratie participative en Europe tandis que les opposants locaux défendent leur espace du quotidien. Les citoyens semblent principalement préoccupés par le chantier de la gare demandant plus de dix ans de travaux dans le centre-ville de Stuttgart et ses environs. Face à la communication faite contre ces grands projets d'aménagement quels sont la stratégie et les outils utilisés par les pouvoirs publics pour défendre et maintenir le projet? 


\section{La mise en place d'une communication officielle par les acteurs publics et privés face à une mobilisation multi-échelles}

À la suite des nombreuses manifestations, parutions d'ouvrages et d'essais remettant en cause Stuttgart 21, les acteurs publics ont décidé de mettre en place une grande campagne de communication. "L'Union pour le projet ferroviaire Stuttgart-Ulm » a créé en 2011 un bureau de communication. Cette instance a pour but de dialoguer avec les médias, d'informer la population sur les travaux en cours et à venir et de s'occuper de la gestion du site internet sur lequel se trouve aussi bien l'historique du projet que les modifications de circulation dans la gare durant les travaux. Des affiches ont également été mises en place dans les halls de la gare centrale de Stuttgart afin d'indiquer les voies aux usagers (Photo 3). La Deutsche Bahn utilise, depuis 1994, la taupe comme symbole de la présence de chantiers dans les gares. Dans le cas de Stuttgart, Max la taupe marque la mise en souterrain des voies de chemin de fer et arbore un air jovial tandis que les passagers peinent à se repérer dans la gare (Stuttgarter Zeitung, 14 septembre 2014).

Photo 3 : Communications sur les travaux en cours dans le quartier de la gare centrale de Stuttgart

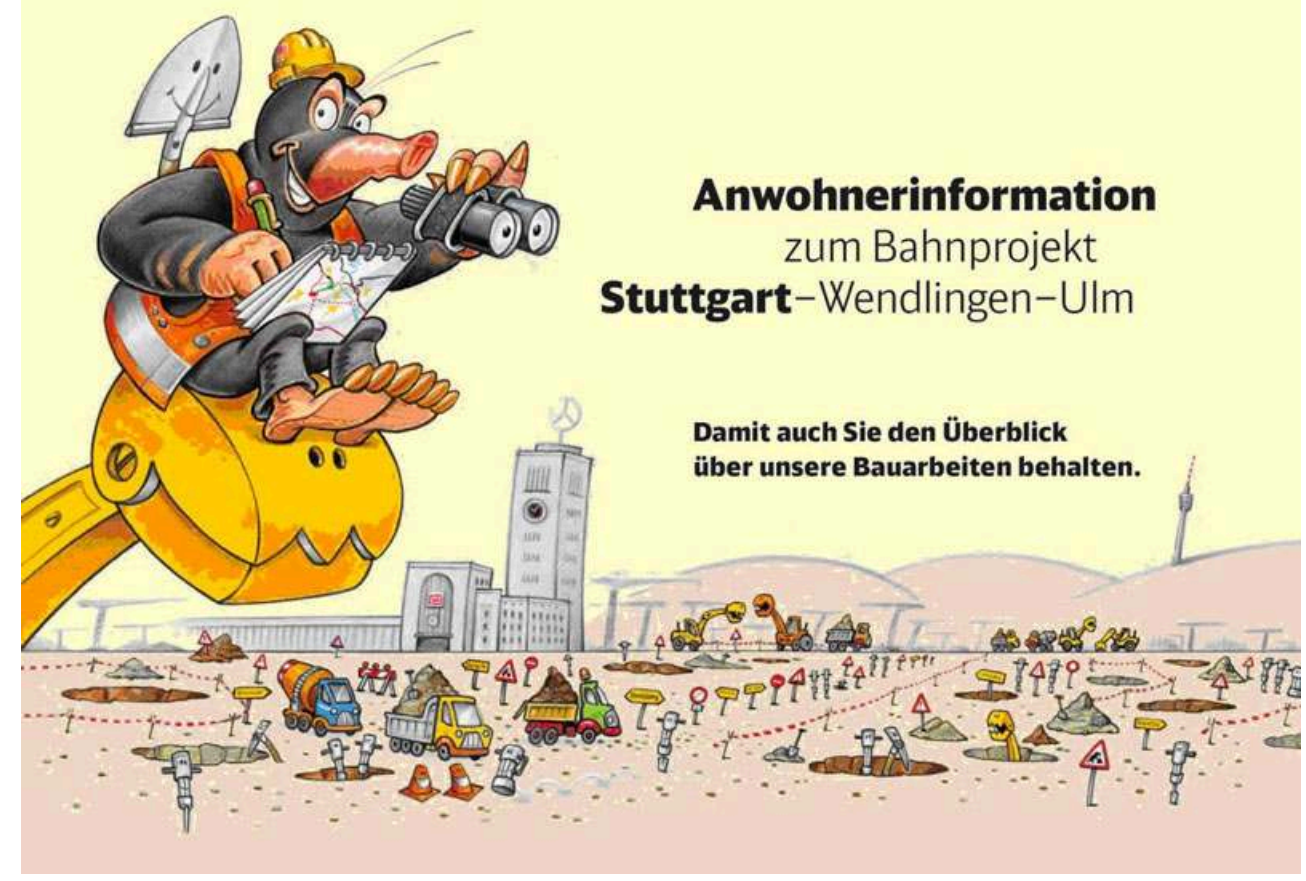

Source : Bahnprojekt Stuttgart-Ulm Verein 


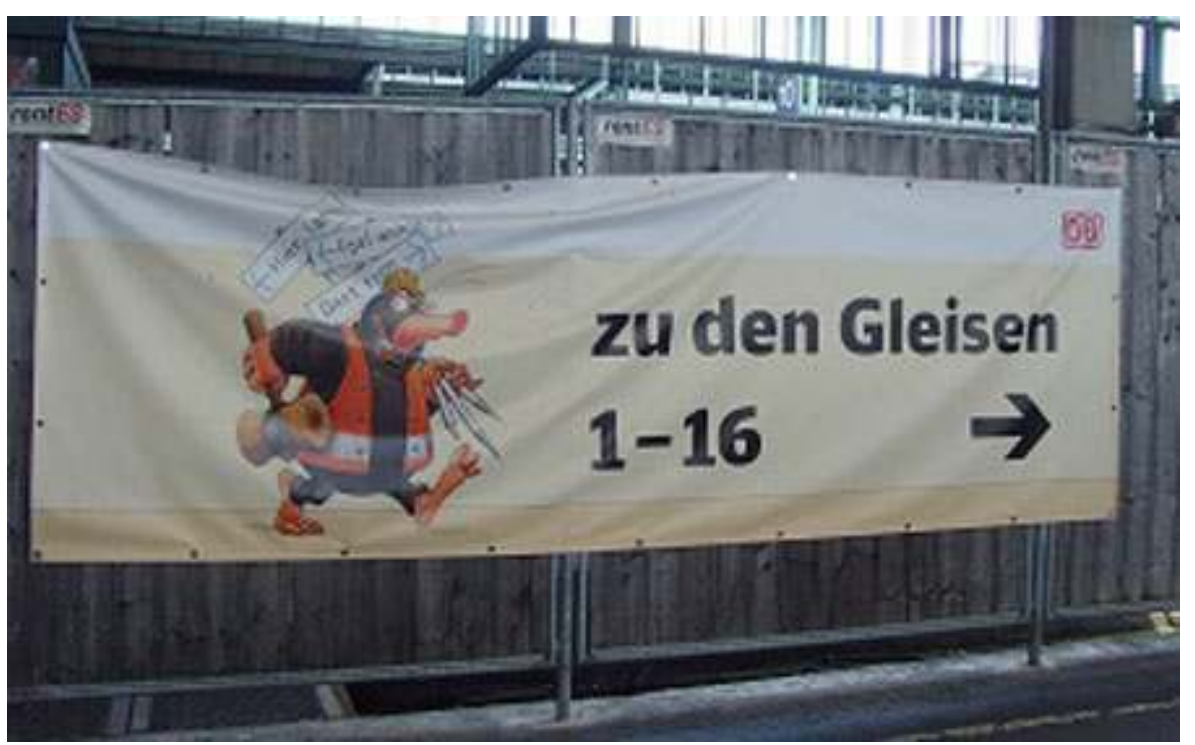

Source : (C)Auteur, 2014

A cela s'ajoute la création en décembre 2011 d'un « Forum pour les citoyens » dédié au projet Stuttgart 21(Bürgerforum S21), sur le conseil de Heiner Geißler (médiateur du projet). Avec le passage de la direction du Land du BW de la CDU à la coalition die Grünen-SPD, les nouveaux acteurs politiques souhaitent regagner la confiance des habitants et donner les moyens de comprendre ce projet qui transforme la ville. L'objectif est de développer le dialogue entre les citoyens et les experts via l'organisation de forums de discussions. Une conférence est par exemple organisée en juin 2012 sur l'utilisation du bois coupé dans le parc du château ${ }^{35}$. Le troisième et dernier outil développé en vue d'informer les habitants se trouve dans le hall de la gare centrale de Stuttgart. La tour d'information Stuttgart 21 comprend dix étages ouverts au public tous les jours et gratuitement ouverte depuis 1998. Cette tour fait partie du bâtiment historique de la gare et permet de valoriser ce symbole précurseur du mouvement de la modernité européenne (europäische Moderne) avec une architecture qui se veut éternelle et qui apparaît, aux yeux de tous, comme un monument du patrimoine architectural de Stuttgart ${ }^{36}$. La ville a été en partie détruite pendant la Seconde Guerre mondiale et conserve peu de monuments antérieurs à 1945, la gare constitue ainsi un élément important de l'architecture de la ville.

La présence, au cœur de l'espace concerné par les travaux, des acteurs porteurs du projet souligne l'importance de la légitimation du projet face à la contestation. Les partenaires publics et privés du projet sont mis en exergue sur les supports de communication. Les plaquettes de présentation de Stuttgart 21 ou de la ligne Wendlingen-Ulm indiquent que "Ce projet a été cofinancé par l'Union européenne réseaux transeuropéens de transport $\aleph^{37}$. D'un côté, il apparaît nécessaire que l'UE soit mentionnée étant donné qu'elle cofinance le projet, ce qui justifie par la même la poursuite des projets sur la section Stuttgart-Ulm. D'un autre côté, la mention de l'UE permet aux acteurs politiques locaux d'invoquer ce soutien pour légitimer son projet. Nous pouvons ici remarquer que les acteurs porteurs du projet des échelles européennes et locales dialoguent et s'entraident directement sans, comme dans la plupart des cas, faire appel à l'échelle nationale. 

porteurs du projet Stuttgart 21 et les habitants, qui pour certains expriment toujours leur désaccord. La 250ème « Manifestation du lundi » a bien eu lieu le lundi 8 décembre 2014 devant la gare centrale.

\section{Conclusion}

La gouvernance du projet global de nouvelle ligne à grande vitesse entre Stuttgart et Ulm apparaît complexe du fait de la multitude d'acteurs en présence. Les différents groupes d'acteurs publics, privés et associatifs qu'ils soient locaux, régionaux, nationaux, européens ou transnationaux expliquent la longue mise en place des décisions et des plans d'actions. Face à la contestation qui continue à Stuttgart, les acteurs publics et privés mettent en œuvre de grandes campagnes de communication dans le but de légitimer ces projets coûteux. $2 / 3$ des fonds destinés à la ligne StuttgartUlm financent en réalité Stuttgart 21, ce qui est à mettre en lien avec l'opposition qui porte précisément sur la rénovation de la gare souhaitée par la Deutsche Bahn et la ville. Les nouveaux acteurs politiques Verts du Land doivent ainsi mettre en place un projet décidé par un autre gouvernement et qui semble difficile à justifier. Cette étude nous montre l'importance prise par la société civile dans les démarches d'aménagement urbain et dans la dénonciation du déficit démocratique en Europe. Les acteurs locaux et européens doivent désormais faire face à mobilisation des citoyens dans la prise de décision. La nouveauté réside, semblerait-il, dans l'expertise des citoyens mobilisés, capables de porter des contre-projets. Ce projet ferroviaire est tout un symbole au pays de Mercedes et de Porsche dans la mesure où les habitants doivent être incités à prendre le train plutôt que la voiture pour les déplacements domiciletravail. Un souhait que formule plus généralement la communauté européenne en privilégiant les transports dits durables. Néanmoins à l'échelle européenne, la question du report modal de l'avion vers le train fait débat et sera mesurée dans les années à venir. La volonté d'européanisation avancée par la Commission européenne est remise en cause par l'abrogation des projets prioritaires t le nouveau découpage de l'axe ParisBratislava en deux tronçons: Paris-Strasbourg et Strasbourg-Bratislava. L'idéal paneuropéen originel pourrait être sorti de son bon rail.

\section{BIBLIOGRAPHY}

ArchitktInnen für K21, 2013, Visionen und Aktionen für Kopfbahnhof und Stadt. Stuttgart, PeterGrohmann, 278p.

Bafoil F., Beichelt T. (dir), 2008 L'européanisation d'Ouest en Est, Paris, l'Harmattan, 327p. Bahnprojekt Stuttgart-Ulm e.V. (2014) Das Bahnprojekt Stuttgart-Ulm, Stuttgart, 23p Balázs P., 2007, Rapport annuel d'activité 2006-2007 pour le PP17, Bruxelles, Commission européenne, 20p.

Revue Géographique de l'Est, vol.55 / n³-4 | 2015 
Balázs P., 2008, Rapport annuel d'activité 2007-2008 pour le PP17, Bruxelles, Commission européenne, $39 \mathrm{p}$.

Balázs P., 2013, Rapport annuel d'activité 2012-2013 pour le PP17, Bruxelles, Commission européenne, $23 p$.

Bilger C., 2014, "Wasserwerferprozess : Polizist berichtet vom Chaos im Park », Stuttgarter Zeitung (en ligne), mis en ligne le 10 septembre 2014, consulté le 21 septembre 2014 URL: http:// www.stuttgarter-zeitung.de/inhalt.wasserwerferprozess-polizist-berichtet-vom-chaos-im-park. 22cdac6e-d177-45f0-89af-7b1e0beefca7.html

Braun S., 2010, «Die Kanzlerin der Attacke », Süddeutsche Zeitung (en ligne), mis en ligne le 23 novembre 2010, consulté le 7 juillet 2014, URL: http://www.sueddeutsche.de/politik/merkelsverwandlung-die-kanzlerin-der-attacke-1.1027417

Braun T., Raidt E., 2010, « Schlossgarten: Wassenwerfer gegen Baumretter », Stuttgarter Zeitung (en ligne), mis en ligne le 17 septembre 2014, consulté le 21 septembre 2014, URL: http:// www.stuttgarter-zeitung.de/inhalt.schlossgarten-wasserwerfer-gegen-baumretter. 63fab5db-1875-4c36-92ac-b540eb2aefa6.html

Brun M., 2013, «Stuttgart 21 : un projet ferroviaire vivement contesté » Suite 21, mis en ligne le 8 juillet 2013, consulté le 30 juin 2014, URL: http://suite101.fr/article/stuttgart-21--un-projetferroviaire-vivement-conteste-a32770\#.U7GFO_1_tBl

Brunet R., 1991, Vers des réseaux transeuropéens. Montpellier, GIP Reclus, 15p.

Bury M., 2012, «Leben in Stuttgart: der Mangel an Wohnungen wird größer ", Stuttgarter Zeitung (en ligne), mise en ligne le 24 septembre 2012, consulté le 28 septembre 2014, URL: http:// www.stuttgarter-zeitung.de/inhalt.leben-in-stuttgart-der-mangel-an-wohnungen-wird-groesser. 00304e5c-073e-4733-a374-b743316f8b93.html

Caron F., Auphan E., 2008, «Vitesses et temps ferroviaires. Un chapitre du programme scientifique de l'AHICF 2008-2013.» Revue d'histoire des chemins de fer, n³9, p.103-109.

Cinotti E., Tréboul JB., 2000, Les TGV Européens. Paris, Presses Universitaires de France. 127p.

Commission européenne, 1992, Livre blanc sur le développement futur de la politique des transports, Rapport Commission européenne, Bruxelles, 90p.

Commission européenne, 2005, Réseau Transeuropéen de Transport. RTE-T, axes et projets prioritaires 2005. Rapport Commission européenne, Office des publications officielles des communautés européennes, 71p.

Commission européenne, 2011a, RTE-T et mécanisme pour l'interconnexion en Europe. Rapport de la Commission européenne, Bruxelles, 57p.

Commission européenne, 2011b, Livre blanc. Feuille de route pour un espace unique des transports vers un système de transport compétitif et économe en ressources. Rapport de la Commission européenne, Bruxelles, 34p.

Commission européenne, 2013a, TEN-T Days EU corridors, backbone for transport in Europe, Vidéo de la Commission européenne, 2min et 5sec, URL: http://ec.europa.eu/transport/themes/ infrastructure/index_en.htm

Commission européenne, 2013b, The core networks corridors - Trans european transport network 2013. Rapport de la Direction mobilité et transport, Bruxelles, 43p. 
Commission européenne, 2014,

Construire le réseau central dans le domaine des transports : corridors de réseau central et mécanisme pour l'interconnexion en Europe. Communication de la Commission, Bruxelles, 13p.

Conseil de l'Union européenne, 1990, Le développement du réseau européen de trains à grande vitesse. Résolution du conseil, Bruxelles, 17 décembre 1990

Damien M-M., 1999 La politique européenne des transports. Paris, Presses universitaires de France, $127 \mathrm{p}$.

Debrie J., Comtois C., 2010. «Une relecture du concept de corridors de transport : illustration comparée Europe/Amérique du Nord.» Les cahiers scientifiques du transport, n58, p.127-144.

Degryse C., 2007, Dictionnaire de l'Union européenne. Bruxelles, De Boeck, 1060p.

Delage A., 2013, La gare, assurance métropolitaine de la ville post-industrielle, Thèse de géographie, Lyon 2, 542p.

Deront E., 2013, « En Allemagne, la bataille de la gare de Stuttgart continue.» Reporterre (en ligne), mis en ligne le 23 janvier 2013, consulté le 30 juin 2014, URL : http://www.reporterre.net/ spip.php?article3756

Dryef Z., 2011, « à Stuttgart, on pense que le progrès ne fait pas le bonheur. » Rue89 (en ligne), mis en ligne le 22 avril 2011, consulté le 1 septembre 2014, URL: http://www.rue89.com/ planete89/2011/04/22/a-stuttgart-on-pense-que-le-progres-ne-fait-plus-le-bonheur-200746

Dubois S., 2010, « TGV : un quart de siècle de bouleversements géoéconomiques et géopolitiques.» Géoécononie, n52, p.89-104.

Equipe de coordination $3^{\text {ème }}$ Forum GPII, 2013, « Le forum européen contre les grands projets inutiles et imposés aura lieu à Stuttgart en juillet.» Reporterre (en ligne), mis en ligne le 22 mai 2013, consulté le 30 juin 2014, URL : http://www.reporterre.net/spip.php?article4282

Fontaine Vive P (de), 2004, « Les grandes infrastructures ferroviaires, leviers de l'intégration européenne.» La revue Transports, n426, p.217-221.

Götz von Olenhusen A., Paulus G., 2010, Oben bleiben! Manifeste und Bilder des Protests. Zürich, Klein und Aber, 207p.

Häußler J., 2011, Dagegen leben? Der Bauzaun und Stuttgart 21: Katalog zur Sonderausstellung 16 Dezember 2011 bis 1. April 2012, Stuttgart, Haus der Geschichte Baden-Württemberg, 212p.

Heuschele H-M., 1970, « Ein Bahnhof unter dem Bahnhof », Stuttgarter Nachrichten, Ed. Du 27 août 1970, p17.

Jünemann H., 2006, Das Projekt Stuttgart 21 und die Neubaustrecke Wendlingen-Ulm. Stuttgart, Turmforum Stuttgart 21, 95p.

Kappus M., 2011, Bürger, Macht, Politik: der Protest gegen Stuttgart 21 als Chance für die Demokratie. Karlsruhe, Von-Loeper Literatur, 115p.

Kost, A., Rellecke, W., Weber, R. (dir.), 2010, Parteien in den deutschen Ländern. Munich, Verlag C.H.Beck, 457p.

Leheis S., 2012, « High-speed train planning in France: Lessons from the Mediterranean TGVline », Transport policy, $n^{\circ} 21, \mathrm{p} 37-44$.

Lepesant G., 2011, Géographe économique de l'Europe centrale. Paris, Les Presses Sciences Po, 351p. 
Lolive J., 1999, Les contestations du TGV Méditerranée. Projets, controverses et espace public. Paris, l'Harmattan, 314p.

Lösch V., Stocker G., Leidig S., Wolf W. (dir), 2011, Stuttgart 21 oder: wem gehört die Stadt? Cologne, Papyrossa, 199p.

Mayet R., 2005, « Le réseau transeuropéen de transport : le dessous des cartes.» Revue du marché commun et de l'Union européenne, $\mathrm{n}^{\circ} 484, \mathrm{p} .11-18$.

Melé P., 2008, « Conflits et controverses : de nouvelles scènes de production territoriale» in Garat I., Séchet R., Zeneidi D. (dir) Espaces en (trans)action, Rennes, PUR, p.239-250.

Novy J., Peters D., 2012, « Railway station in mega-projects as public-controversies: the case of Stuttgart $21 »$, Built Environment, Vol 38, n²1, p.128-145.

Perron C., 2011, «Stuttgart 21 : un projet qui met en danger la stabilité du gouvernement allemand.» Ceri-Sciences-Po (en ligne), mis en ligne février 2011, consulté le 30 janvier 2014, URL: http://www.sciencespo.fr/ceri/fr/content/stuttgart-21-un-projet-qui-met-en-danger-lastabilite-du-gouvernement-allemand

Perron C., 2012, Le Baden-Württemberg : un modèle? Paris, Coesionet, 54p.

Reiser W., 2011, Die ganze Wahrheit über Stuttgart 21: und wie es mit uns allen weitergeht. Munich, Scorpio, 189p.

Rey V., Groza O., « Bulgarie et Roumanie, une affaire d'européanisation.» L'Espace géographique, Tome 37, nº 4 , p.289-296.

Schmierer S., 2014, « Ortsbesuch im Stuttgarter Hauptbahnhof: wo, bitte, geht's hier zum ICE? », Stuttgarter Zeitung (en ligne), mis en ligne le 14 septembre 2014, consulté le 29 septembre 2014, URL: http://www.stuttgarter-zeitung.de/inhalt.ortsbesuch-im-stuttgarter-hauptbahnhof-wobitte-geht-s-hier-zum-ice.ae990ffb-96c9-4909-9083-11fd21744833.html

Scholl B., 2014, «Integrated spatial und infrastructural development: the need for adequate methods and spatial strategies for collaborative action and decision-making ", p.63-83., In Analytical decision-making methods for evaluation sustainable transport in European corridors, Lami I., Schorlau W., 2010, Stuttgart 21: die Argumente. Cologne, Kiepenheuer und Witsch, 266p.

Schunder J., 2013, « Wohnungsnot in Stuttgart », Stuttgart Nachrichten (en ligne), mis en ligne le 6 décembre 2013, consulté le 29 septembre 2014, URL: http://www.stuttgarter-nachrichten.de/ inhalt.wohnungsnot-in-stuttgart-kuhn-will-sich-mit-wohnungsleerstand-nichtabfinden.bde908eb-af37-461f-a985-c467b15c1567.html

Siarov V., 2004, Evaluation stratégique des infrastructures de transport. Thèse de géographie, Paris 8 , $704 \mathrm{p}$.

Sichelschmidt H., « The EU programme Trans-european network - a critical assessment », Transport Policy, Vol. 6, n³, p.169-181.

Silla O., 2006, « Des coordonnateurs pour le réseau transeuropéen de transport.» Revue du marché commun et de l'Union européenne, $\mathrm{n}^{\circ} 494, \mathrm{p} .42-46$.

Spiegel Online, 2010, « Geißlers Schlichtung: sieben Punkte Plan soll Stuttgart 21 retten », mis en ligne le 30 novembre 2010, consulté le 1 septembre 2014, URL: http://www.spiegel.de/politik/ deutschland/geisslers-schlichtung-sieben-punkte-plan-soll-stuttgart-21-retten-a-732008.html 
Statistisches Landesamt BW, 2013, Berufspendler in Baden-Württemberg, Rapport du Statistisches Landesamt BW, mis en ligne en septembre 2013, consulté le 22 septembre 2014, URL: http:// www.fafobw.de/Veroeffentl/Statistik_AKTUELL/803413009.pdf\#search=berufspendler

Treiber A., 2013, « Chronologie zu Stuttgart 21 : Schwarzer Donnerstag im Schlossgarten », Stuttgarter Zeitung, mis en ligne le 7 décembre 2013, consulté le 21 septembre 2014, URL: http:// www.stuttgarter-zeitung.de/inhalt.chronologie-zu-stuttgart-21-schwarzer-donnerstag-imschlossgarten.114e1e4e-5f61-4523-8f8c-9a387733c0ed.html

Union européenne, 1992, Traité de Maastricht. Journal officiel de la Communauté européenne, $115 p$

Varlet J., 2000, « Dynamique des interconnexions des réseaux de transports rapides en Europe : devenir et diffusion spatiale d'un concept géographique », Flux, n41, p.5-16.

\section{Sites internet consultés}

Bahnprojekt Stuttgart-Ulm, www.bahnprojekt-stuttgart-ulm.de, consulté le 19 septembre 2014

Direction Mobilité et transport de la Commission européenne, www.ec.europa.eu/transport, consulté le 9 juillet 2014

Magistrale pour l'Europe, www.magistrale.org/fr/startseite.html, consulté le 18 juillet 2015

\section{NOTES}

1. Conseil européen qui s'est déroulé à Essen (Allemagne) en 1994

2. Dix-huit axes sur les trente comprennent du transport ferroviaire

3. La contribution de l'UE dans le cadre du transport ferroviaire est répartie comme suit : $50 \%$ maximum d'aide pour les études, $40 \%$ maximum d'aide à la réalisation des sections avec tronçon transfrontalier, 30\% maximum d'aide à la réalisation des sections avec goulet d'étranglement, $20 \%$ d'aide à la réalisation pour les autres sections (Commission européenne, 2011a).

4. «transforming a patchwork into a network » in Commission européenne, 2013; URL : http:// ec.europa.eu/transport/themes/infrastructure/index_en.htm

5. "one of the key east-west oriented TEN-T links in Europe" in "Direction mobilité et transport" de la Commission européenne, URL : http://ec.europa.eu/transport/themes/infrastructure/tent-policy/linking_en.htm

6. Données Eurostat 2013, régions entre 100 et 500 hab. $/ \mathrm{km}^{2}$, URL: http://ec.europa.eu/ eurostat/statistical-atlas/gis/viewer/?chapter $=02$

7. Excepté entre avril 2009 et mai 2010 où il a été ministre des affaires étrangères du gouvernement Bajnai

8. Fonds européens mobilisés : fonds RTE-T, Plan de relance de l'économie européenne et le fonds de cohésion pour lequel la Slovaquie est éligible

9. Consulter le site internet de la Magistrale pour l'Europe - Magistrale für Europa : http:// www.magistrale.org/fr/startseite.html

10. Le Fils est un affluent du Neckar lui-même affluent du Rhin

11. Entretiens avec des universitaires allemands, Stuttgart, 2015

12. Données provenant du site Bahnprojekt Stuttgart-Ulm, URL: http://www.bahnprojektstuttgart-ulm.de/details/kosten-und-finanzierung/

13. Deutsche Bahn SA, Deutsche Bahn Netz SA, Deutsche Bahn Station und Service

14. Exposition à la Turmforum Information située dans le hall de la gare centrale de Stuttgart 
15. Il n'existe pas de liaison ferroviaire directe entre Ulm et l'aéroport de Stuttgart. Les voyageurs doivent se rendre à la gare centrale de Stuttgart (environ $1 \mathrm{~h}$ ) puis se rendre avec le SBahn jusqu'à l'aéroport

16. Bahnprojekt Stuttgart-Ulm Kommunikationsbüro

17. „Jetzt geht's los! Stuttgart 21 kommt. Baustart 2. Februar 2010“

18. CDU : Christlich Demokratische Union Deutschland (Union chrétienne-démocrate d'Allemagne)

19. SPD : Sozialdemokratische Partei Deutschland (parti social-démocrate d'Allemagne)

20. FDP : Freie Demokratische Partei (parti libéral démocrate)

21. Die Grünen : Parti Vert

22. Lire à ce sujet : Perron, 2012 ; Kost, A ; Rellecke, W ; Weber, R (dir.). (2010)

23. Le financement des travaux du projet Stuttgart 21 sont en partie financés par la vente des espaces actuellement occupés par les voies ferrées ainsi que par un dépôt ferroviaire (Nordbahhofviertel). La Deutsche Bahn a vendu ces surfaces à la ville de Stuttgart qui en est donc propriétaire.

24. Vidéo de présentation du projet; Url : https://www.youtube.com/watch?v=rbM0RtOXmxI

25. Entretien au MVI du Land du BW, Stuttgart, juillet 2014

26. La construction de voiture est le premier pôle d'emplois dans la ville de Stuttgart Statistisches Landesamt Baden-Württemberg,

URL : http://www.statistik.baden-wuerttemberg.de/Veroeffentl/Statistik_AKTUELL/ 803413007.pdf

La voiture est le premier moyen de transport utilisé pour les trajets domicile-travail

Statistisches Landesamt Baden-Württemberg,

URL : http://www.fafobw.de/Veroeffentl/home.asp?P=A\&A=\&S=\&T=\&V=\&B=

27. Données de la Deutsche Bahn sur le site site Bahnprojekt Stuttgart-Ulm, URL: http:// www.bahnprojekt-stuttgart-ulm.de

28. Accès aux fonctions récréatives et commerciales de la ville (ouverture du centre commercial Milanéo à l'automne 2014)

29. Statistisches Landesamt Baden-Württemberg,

URL : http://www.statistik.baden-wuerttemberg.de/Veroeffentl/Statistik_AKTUELL/ 803413007.pdf

30. Entretiens avec des universitaires allemands, Stuttgart, 2014 et 2015

31. Schloßgarten attenant à la gare centrale de Stuttgart

32. Il est indiqué p4 que lorsque les travailleurs se rendent à leur travail depuis leur domicile $50 \%$ utilisent leur voiture (s'ils habitent et travaillent dans la même commune) contre $83 \%$ (si la commune de résidence est différente de la commune de travail), Statistisches Landesamt BadenWürttemberg

URL : http://www.fafobw.de/Veroeffentl/home.asp?P=A\&A=\&S=\&T=\&V=\&B=

33. Ancien secrétaire général de la CDU et membre de l'organisation ATTAC

34. Déclaration élaborée par des associations et mouvements qui luttent contre la construction de grands projets d'infrastructures

35. Workshop „Verwendung des Holzes aus dem Schloßgarten“

36. Entretiens avec des universitaires allemands, Stuttgart, 2015

37. Plaquette de présentation du Bahnprojekt Stuttgart-Ulm «Dieses Projekt wird von der europäischen Union kofinanziert - Transeuropäische Netze für Vekehrsinfrastrukturen » 


\section{ABSTRACTS}

In 2004 , the European commission decided to support thirty priority projects concerning transport infrastructure. The project $n^{\circ} 17$ aims to establish a high-speed rail line between Paris and Bratislava. This line will cross four countries (France, Germany, Austria and Slovakia), different regions and cities. In the case of the European transport reform, the priority projects have been replaced in January 2014 by the transeuropean corridors in order to create a single European area. The high number of people engaged in the governance complicates and slows the beginning of the project. The railway line Paris -Bratislava has several development projects around railway stations (construction or extension) including the controversial project Stuttgart 21. The German capital city of Baden-Württemberg and Deutsche Bahn have, in fact, since the 1980s, the desire to renovate Stuttgart Central station. The dispute has intensified in 2010 with the start of work. It is mainly localized in Stuttgart but we also found the presence of national or transnational actors alongside residents. Who are the targeted actors of the contestation? How the different decision and action scales join in order to try to raise the project?

La décision de la Commission européenne de mettre en place trente projets prioritaires en 2004 a conduit à l'élaboration d'une ligne ferroviaire à grande vitesse entre Paris et Bratislava. Le projet prioritaire $n^{\circ} 17$ traverse quatre États de l'Union européenne (France, Allemagne, Autriche et Slovaquie) aux structures territoriales différentes. Dans le cadre d'une réforme de la politique des transports, les projets prioritaires ont été remplacés depuis janvier 2014 par les corridors transeuropéens afin d'obtenir un maillage unifié de l'espace européen. La gouvernance demeure complexe du fait du grand nombre d'instances participant à l'élaboration des projets, ce qui explique la longueur de leur mise en place. La ligne ferroviaire Paris-Bratislava comprend plusieurs projets d'aménagement autour des gares (construction ou extension) parmi lesquels le projet controversé Stuttgart 21. La ville-capitale du Land allemand du Bade-Wurtemberg ainsi que la Deutsche Bahn ont, en effet, depuis les années 1980, le souhait de rénover la gare centrale de Stuttgart. La contestation s'est accentuée en 2010 avec le début des travaux. Elle s'est principalement localisée à Stuttgart mais nous avons également pu constater la présence d'acteurs nationaux voire transnationaux aux côtés des habitants. Quels sont les acteurs visés par la contestation ? Comment, face aux oppositions, les différentes échelles de décisions et d'actions s'entremêlent pour essayer de faire émerger ce projet?

Im Jahr 2004 beschloss die Europäische Union dreißig Prioritätsprojekte um die Verbesserung der Verkehrsinfrastruktur $\mathrm{zu}$ schaffen. Das Projekt Nr.17 verbindet vier Staaten der europäischen Union (Frankreich, Deutschland, Österreich und die Slowakei) mit verschiedenen räumlichen Strukturen. Im Rahmen einer verkehrspolitischen Reform um die Vernetzung des europäischen Raums zu ermöglichen, wurden die europäischen Prioritätsprojekte in Januar 2014 durch den transeuropäischen Korridor ersetzt. Die Governanz ist jedoch komplex, da es viele Instanzen gibt, die an der Schaffung der Projekte beteiligt sind. Dies führt zu einer langwierigen Umsetzung der Projekte. Die Eisenbahnlinie Paris-Bratislava umfasst mehrere Entwicklungsprojekte rund um verschiedene Bahnhöfe (Bau oder Erweiterung), darunter das umstrittene Projekt Stuttgart 21. Die deutsche Hauptstadt von Baden-Württemberg und die Deutsche Bahn haben schon seit den 80er Jahren den Wunsch, den Stuttgarter Hauptbahnhof zu renovieren. Der Streit um das Bauprojekt hat sich im Jahr 2010 mit dem Beginn der Arbeiten intensiviert. Der Konflikt wird vor allem in Stuttgart auf lokaler Ebene ausgetragen, aber neben den Einwohnern sind auch weitere nationale oder transnationale Akteure daran beteiligt. An 
welche Akteure richtet sich der Protest? Wie sind die verschiedenen Beschlussebenen und Aktionen miteinander verbunden, um dieses Projekt zu realisieren?

\section{INDEX}

Keywords: contestation, Europe, European priority projects, European Union, Germany, governance, high-speed rail system, Stuttgart, Stuttgart 21, Transeuropean corridors

Schlüsselwörter. der Protest, Deutschland, die Governanz, Europa, Europäische Union, europäisches Prioritätsprojekt, Hochgeschwindigkeitstreckennetz, Stuttgart, Stuttgart 21, transeuropäischer Korridor

Mots-clés: Allemagne, contestation, corridors transeuropéens, Europe, gouvernance, grande vitesse ferroviaire, projets prioritaires européens, Stuttgart, Stuttgart 21, Union européenne

\section{AUTHOR}

\section{ANAÏS VOLIN}

Doctorante à l'Université Lumière Lyon 2, UMR 5600 Environnement Ville Société - ENS de Lyon, 15 Parvis René Descartes 69007 Lyon - anais.volin@univ-lyon2.fr 